\begin{tabular}{c|c|c}
$\begin{array}{c}\text { Military Technical College } \\
\text { Kobry El-Kobbah, } \\
\text { Cairo, Egypt }\end{array}$ & $\begin{array}{c}8^{\text {th }} \text { International Conference } \\
\text { on Electrical Engineering } \\
\text { ICEENG 2010 }\end{array}$
\end{tabular}

\title{
Environment Impact Assessment of Sewage Treatment Plants al Case Study AL-Berka Sewage Treatment Plant
}

\author{
By \\ N. Saoud, * \\ M. Fergala, ** \\ N.Abdel Mutaal***, N.Amer****
}

\section{Abstract:}

The present research aims to the environmental impact assessment of the treatment plant of Al-Berka and studying the causes of the environmental deterioration and emphasizing enhancement of activities. It is recognized that in order to achieve sustainable and optimal development, it is essential to ensure that environmental impact assessment is undertaken. Such assessment should ensure that there is an optimal amount of information about the key issues that should be addressed. Wise decision-making, which is necessary for sustainable development, can only occur if as much information as possible about the likely effects of a given development. The present research results indicated that the treatment plant Al-Berka in application case of not applying mitigation measures during on and from the viewpoint of environmental impact gave weights percentage $(+29.42 \%)$ and in case of applying mitigation measures, the weights percentage raised to $(+53.23 \%)$. Enhancement of $(81 \%)$ was calculated. The worst environment aspects were odor and sludge. Best methods to avoid environmental odor impact are active carbon pumped in the project site. It helps and oxidizes and gives results after application due to gases adsorption in treatment plant Al-Berka. Sludge treatment technique used offers a reduction of $(95 \%)$ of pollution causes, due to preventing soil pollution and the harmful materials oxides. Odor represents one of the most environmental aspects that have impact on the environment and the mitigation applied by pumping carbon in the plant site giving a mitigation measure about (weight before mitigation $-81 \%$ after mitigation $+50 \%$ ). Sludge generated from treatment process has a significant negative impact on the environmental(weight before mitigation $-48 \%$ ). This causes environmental problems to air, soil, groundwater, surface water and public health. The mitigation gives an enhancement (weight after mitigation $+66 \%$ ). An improvement of $(+9.4 \%)$ above baseline conditions, is considered as remarkable positive impact on the socio-economic dimension. A remarkable improvement on the 
political impact dimension scores $(28.6 \%)$ above baseline condition. The main reasons are the social acceptance of the project and the enhancement of government image as a result of implementing the project as well as the willingness to apply mitigation measures in the long run.

\section{Keywords:}

Environment Impact Assessment, Sewage Treatment.

*Syrian Armed Forces

*. Col.Research. Eng. Noaman Hasan Saoud-(Ph.D student) Military Technical College

**.Prof. Dr. Mohamed A. Fergala Ain Shams University Faculty of Engineering

***.Maj. Gen. Dr. Nabil. Husean Abdel-Mutaal Military Technical College

**** Brig. Dr. Nabil Hasan Amer Military Technical College

\section{Introduction:}

\subsection{General:}

The EIA process has evolved into a series of stages. It is not suggested that the process described below is followed in all circumstances, but is the distillation of experiences from many countries over the last 20 years. Difference in environmental, legislative, political and technical circumstances will often require modifications to the process to be made. Below, are described the objectives of each

Stage and their relationship to the process as a whole:

Definition of the proposal: The design and selection of the preferred alternative.

Screening: The determination of which projects will require a full EIA.

Scoping: The process of identifying a number of priority issues from the broad range of potential challenges, and focusing the assessment on a manageable number of important questions.

Assessment of impacts: The process of determining the magnitude and the importance of changes that are expected to result.

Stages of the EIA process (Mitigation and enhancement, Review, Monitoring, Impact auditing [Alternatives: Development option], No development option, Examine 
different solutions, Alternatives sites and corridors, Design options, Different designs and layouts).Typically, sewage treatment involves three stages called primary, secondary and tertiary treatment; the primary treatment consists of two steps: the first step in wastewater treatment called preliminary treatment which separate the debris, sticks, rags, large food particles, sand, gravel, toys, etc. Which are removed at this step to protect the pumping and other equipment in the treatment plant. In the second step performances the separates suspended solids, oils and greases from wastewater. In secondary treatment a biological treatment process is conducted to remove dissolved organic matter from wastewater. But in the final treatment focuses on removal of disease-causing organisms from wastewater. Treated sewage can be disinfected by adding chlorine or others chemical treated.

The increase in cell number is accompanied by oxygen production during photosynthesis which aids the rapid aerobic decomposition of residual organic matter by bacteria, Also studied the feasibility of growing algae in settled domestic sewage in California with average yields of algae in excess of $100 \mathrm{Kg}$ dry weight / hectar. The liquid phase of pig slurry was used as a medium for algal biomass production by Garrett and Fallow field (1980). They mentioned that by this process, using the alga Chlorella vulgaris, they could remove the polluting nutrients such as phosphorus, reduction of BOD and production of biomass. [1]

Studied the bacterial micro flora in Chlorella vulgaris cultures and in nitrogen containing industrial waste water. They mentioned that the decrease of some bacterial groups occurring during cultivation of algae is due to antibacterial agent produced by chlorella vulgaris. [2]

During growth, algal cells convert dissolved N, P and C to algal protoplasm. They remove these elements from the waste stream, and on harvest of the cells, leave behind a renovated wastewater which can be reused. [3]

They has a vital role to play in transforming sewage and wastewater into valuable bio-mass and treated water can be used for irrigation. These advantages have also generated interest in recycling the domestic and rural wastes through algal systems. [4]

Observed that the temperature affects the solubility limitation of oxygen required for aerobic treatment of wastewater stated that an increase of temperature decreases the wastewater viscosity and its surface tension resulting in improved mixing and biochemical reaction. Also, a high temperature increases the chemical and biological activity of the process resulting in the need for large treatment plant component.[5]

\section{Description of the plant:}

Description of the plant: A $600,000 \mathrm{~m} 3 / \mathrm{d}$ wastewater treatment plant of AL-BERKA was being designed to treat generated domestic and industrial wastewater. The design of the treatment facility as a primary and secondary treatment steps is capable of eliminating the organic and microbial pollutants to the allowable extents.

Plant site: Al-Salaam district city. 
-Treated type: primary and secondary, treatment. Primary started on November 1990 while the secondary treatment started on March 1998.

-Station cost of construction: 360 million pounds, while operation cost and annual maintenance: about million.

-Project capacity design plant: $600000 \mathrm{~m}^{3} /$ day,

-Operation capacity about: $500000-550000 \mathrm{~m}^{3} /$ day,

-Annual removal ratio: BOD mg/l (influent: 475 - effluent: 24 - efficiency 94\%,

-TSS .mg/L (influent: 500 - effluent: 30 - efficiency: 93\%),

-Liquid sludge (amount: $5670 \mathrm{~m} 3$ / day),

-Dry sludge about $4000 \mathrm{~m} 3$ /day),

-Population served: about 2 million,

-Population design number: about 3 million.

- Station work: good, electric consumption: 2-3 million kilowatts per month,

-Sewage exit: two emergency canals.

-Entrance: spiral station in two stages, 6 pumps each stage (total 12 pumps).

-The project objects: Treatment sewage water biological complete.

Introduced weighting system for environmental impacts:The Weighting is based the foundations of advanced technology, Entrance to an integrated training in scope EIA National Organization for Potable Water and Sanitary Drainage Egyptian. On a plus (+) or minus (-) ten-grade system. A Weighting equal to ZERO for a specific criterion refers to the case of no change from baseline conditions (pre-project conditions). This means that the impact resulted from a certain action within the project context on such a specific criterion will NOT alter the existing environmental conditions. The worst environmental impacts (deterioration) correspond to a minimal weight of $(-10)$, while the best environmental impacts (enhancement) correspond to a maximum score of (+10). Table (1) presents the matrix designed to cover the environmental impacts of the project, in case of not applying measures during construction \& operation, however table (2) presents them in case of applying measures during construction and operation. Details on calculations of the sub-items are listed and presented in tables (1), (2). The final weighted scores reflect the relative importance of the relative aspects as shown in tables(3)\&(4)\&(5)\&(6) below and considering the following:1- Al-Berka treatment plant did not assessed environmentally.

2-The tenth-grade system is used previously in reports submitted to EEAA. This evaluation system has a relative lack in accuracy.

3-The author suggests a modification to the tenth-grade to be based on thousand grade evaluation in order to enhance accuracy, comparison, making decision and improves results. The enhanced results are shown in tables (7).4-As indicated in tables (5) - (6), the accepted weight in Egypt and other different countries ranges $(2 \sim 18)$. 


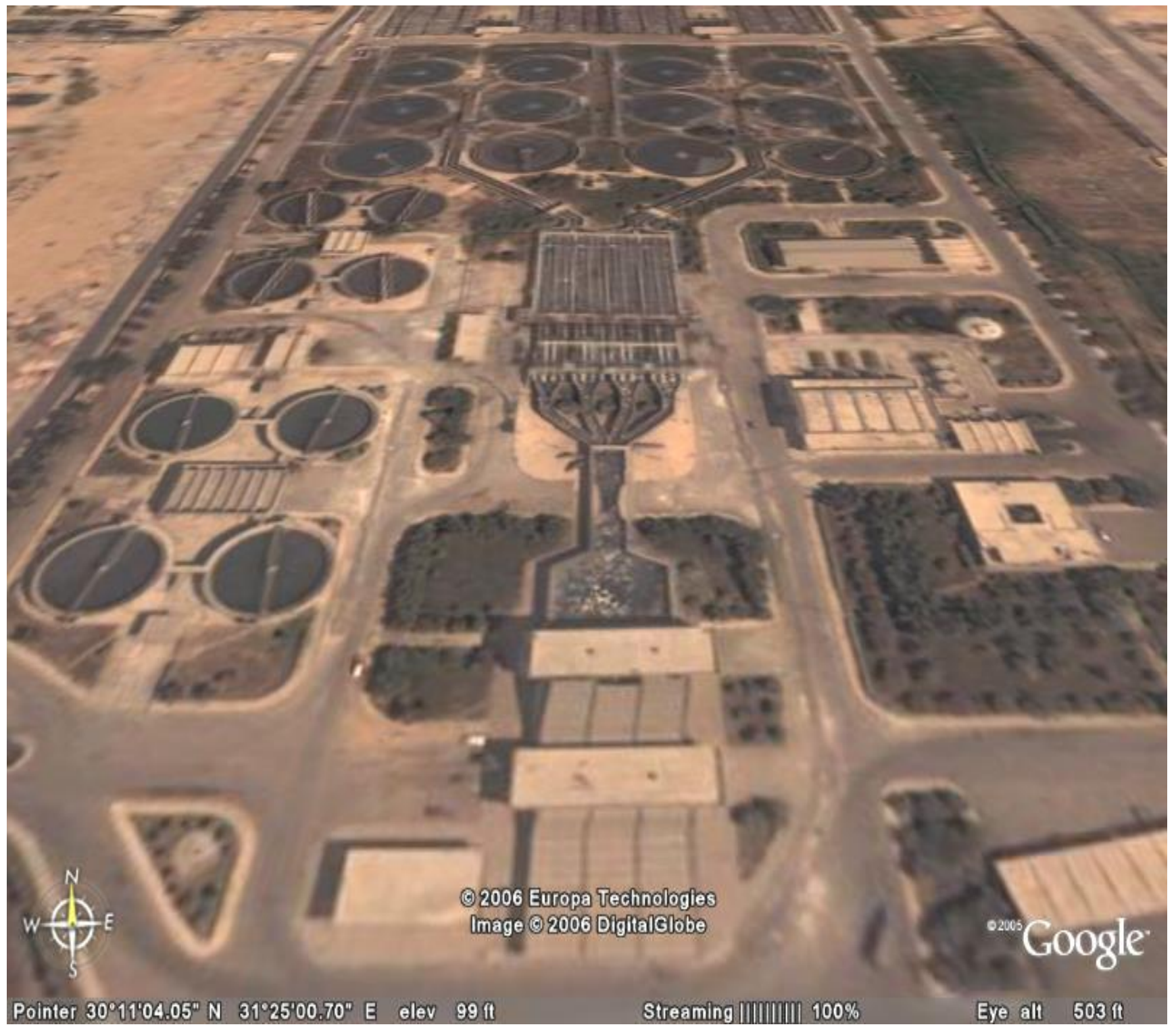

Photo (1) General layout of the treatment plant $f$ the Al-Berka. 
Table (1) Weighted score for environmental impacts during construction \& Operation phases (in case of not applying mitigation measures).

\begin{tabular}{|c|c|c|c|c|c|c|c|}
\hline 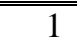 & $\overline{22}$ & 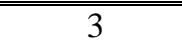 & 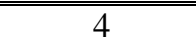 & $\overline{\overline{5}}$ & 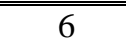 & $\overline{\overline{77}}$ & $\overline{88}$ \\
\hline No. & $\begin{array}{l}\text { Environmental } \\
\text { Component }\end{array}$ & $\begin{array}{c}\text { Cumulative } \\
\text { Score }\end{array}$ & $\begin{array}{l}\text { No. of sub. } \\
\text { Components }\end{array}$ & $\begin{array}{l}\text { Max/Min } \\
\text { Score }\end{array}$ & Score \% & $\begin{array}{c}\text { Weight } \\
\text { Ten-grade }\end{array}$ & $\begin{array}{l}\text { Normalized } \\
\text { Score }\end{array}$ \\
\hline A. & $\begin{array}{l}\text { Natural resources } \\
\text { Impact criteria }\end{array}$ & & & & & & \\
\hline 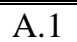 & Soil & 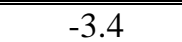 & 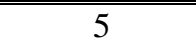 & $\overline{c 50}$ & 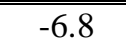 & 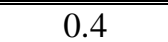 & 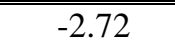 \\
\hline A.2 & Water & $\overline{-0.8}$ & 7 & 70 & $\overline{-1.1}$ & $\overline{0.3}$ & -0.33 \\
\hline A.3 & Air & +3.2 & 3 & 30 & +10.7 & 0.3 & +3.21 \\
\hline & Sub-total & & & & & 1 & +0.16 \\
\hline B. & $\begin{array}{l}\text { Biological life } \\
\text { Impact criteria }\end{array}$ & & & & & & \\
\hline$\overline{B .1}$ & Plant life & +4 & $\overline{c 6}$ & 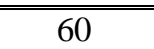 & +6.7 & 0.7 & +4.69 \\
\hline B.2 & Animal life & +5 & 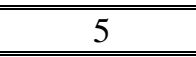 & 50 & +10 & 0.3 & +3 \\
\hline & Sub-total & & & & & 1 & +7.69 \\
\hline C. & $\begin{array}{l}\text { Socio-economic } \\
\text { Impact criteria }\end{array}$ & & & & & & \\
\hline C.1 & General & +2.5 & 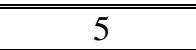 & 50 & +5 & 0.05 & +0.25 \\
\hline C.2 & $\begin{array}{l}\text { Public services and } \\
\text { utilities }\end{array}$ & $\begin{array}{l}-0.1 \\
\end{array}$ & 7 & $\overline{770}$ & 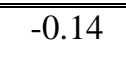 & 0.2 & "-0.028 \\
\hline C.3 & Human health & $\begin{array}{l}-0.1 \\
\end{array}$ & 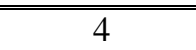 & $\overline{440}$ & 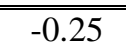 & $\overline{0.2}$ & $\begin{array}{c}-0.05 \\
\end{array}$ \\
\hline C.4 & $\begin{array}{l}\text { Creation of new } \\
\text { Communities }\end{array}$ & +2 & 1 & 10 & +20 & 0.05 & $\overline{+1}$ \\
\hline C.5 & $\begin{array}{l}\text { Solid waste \& } \\
\text { Hazardous waste }\end{array}$ & -2 & 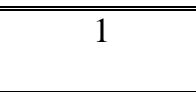 & 10 & -20 & 0.1 & -2 \\
\hline 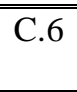 & $\begin{array}{l}\text { NGOs and local } \\
\text { Unions }\end{array}$ & 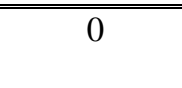 & $\bar{~} 1$ & 10 & 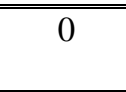 & 0.1 & 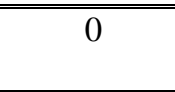 \\
\hline
\end{tabular}

\begin{tabular}{|c|c|c|c|c|c|c|c|}
\hline C.7 & $\begin{array}{l}\text { Sustainability of } \\
\text { Services }\end{array}$ & 0 & 1 & 10 & 0 & 0.2 & 0 \\
\hline \multirow[t]{2}{*}{ C. 8} & $\begin{array}{l}\text { Substantial fuel } \\
\text { or energy consumption }\end{array}$ & -5 & 1 & 10 & -50 & 0.1 & -5 \\
\hline & Sub-total & & & & & 1 & -5.83 \\
\hline D. & $\begin{array}{l}\text { Political impacts } \\
\text { Criteria }\end{array}$ & & & & & & \\
\hline D. 1 & $\begin{array}{l}\text { Public support \& } \\
\text { Participation }\end{array}$ & +0.6 & 1 & 10 & +6 & 0.1 & +0.6 \\
\hline D. 2 & Social acceptance & +0.6 & 1 & 10 & +6 & 0.1 & +0.6 \\
\hline D.3 & $\begin{array}{l}\text { Building trust in local } \\
\text { authorities }\end{array}$ & +0.6 & 1 & 10 & +6 & 0.1 & +0.6 \\
\hline D.4 & $\begin{array}{l}\text { Enhancement of } \\
\text { Governmental Image }\end{array}$ & +0.6 & 1 & 10 & +6 & 0.1 & +0.6 \\
\hline D.5 & $\begin{array}{l}\text { Promotion of } \\
\text { Gender equity }\end{array}$ & 0 & 1 & 10 & 0 & 0.1 & 0 \\
\hline D.6 & $\begin{array}{l}\text { Natural infra- } \\
\text { structural plans } \\
\end{array}$ & +5 & 1 & 10 & +50 & 0.5 & 25 \\
\hline & Sub-total & & & & & 1 & +27.4 \\
\hline & Total & & & & & & +29.42 \\
\hline
\end{tabular}


Table (2) Weighted score for environmental impacts during construction \& Operation phases (in case of applying mitigation measures).

\begin{tabular}{|c|c|c|c|c|c|c|c|}
\hline 1 & 2 & 3 & 4 & 5 & 6 & 7 & 8 \\
\hline No. & $\begin{array}{l}\text { Environmental } \\
\text { Component }\end{array}$ & $\begin{array}{l}\text { Cumulative } \\
\text { Score }\end{array}$ & $\begin{array}{l}\text { No. of sub. } \\
\text { Components }\end{array}$ & $\begin{array}{l}\text { Max/Min } \\
\text { Score }\end{array}$ & Score $\%$ & $\begin{array}{c}\text { Weight } \\
\text { Ten-grade }\end{array}$ & $\begin{array}{l}\text { Normalized } \\
\text { Score }\end{array}$ \\
\hline A. & $\begin{array}{l}\text { Natural resources } \\
\text { Impact criteria }\end{array}$ & & & & & & \\
\hline A. 1 & Soil & +1.35 & 5 & 50 & +2.7 & 0.4 & +1.08 \\
\hline A. 2 & Water & +1.75 & 7 & 70 & +2.5 & 0.3 & +0.75 \\
\hline A.3 & Air & +5.6 & 3 & 30 & +18.7 & 0.3 & +5.6 \\
\hline & Sub-total & & & & & 1 & +7.43 \\
\hline B. & $\begin{array}{l}\text { Biological life } \\
\text { Impact criteria } \\
\end{array}$ & & & & & & \\
\hline B.1 & Plant life & +3.45 & 6 & 60 & +5.75 & 0.7 & +4.03 \\
\hline B. 2 & Animal life & +7.2 & 5 & 50 & +14.4 & 0.3 & +4.32 \\
\hline & Sub-total & & & & & 1 & +8.35 \\
\hline$\overline{\mathrm{C} .}$ & $\begin{array}{l}\text { Socio-economic } \\
\text { Impact criteria }\end{array}$ & & & & & & \\
\hline C. 1 & General & +7 & 5 & 50 & +14 & 0.05 & +0.7 \\
\hline C. 2 & $\begin{array}{l}\text { Public services and } \\
\text { utilities }\end{array}$ & +0.2 & 7 & 70 & +0.2857 & 0.20 & +0.6 \\
\hline C. 3 & Human health & +2.2 & 4 & 40 & +5.5 & 0.20 & +1.1 \\
\hline $\begin{array}{ll}\text { C.4 } \\
\end{array}$ & $\begin{array}{l}\text { Creation of new } \\
\text { Communities }\end{array}$ & +4 & 1 & 10 & +40 & 0.05 & +2 \\
\hline C.5 & $\begin{array}{l}\text { Solid waste \& } \\
\text { Hazardous waste }\end{array}$ & +3 & 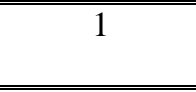 & 10 & +30 & 0.1 & +3 \\
\hline C.6 & $\begin{array}{l}\text { NGOs and local } \\
\text { Unions }\end{array}$ & +2 & 1 & 10 & +20 & 0.1 & +2 \\
\hline C.7 & $\begin{array}{l}\text { Sustainability of } \\
\text { Services }\end{array}$ & $\overline{00}$ & 1 & 10 & 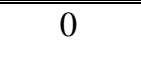 & 0.2 & 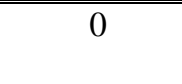 \\
\hline
\end{tabular}

\begin{tabular}{|c|c|c|c|c|c|c|c|}
\hline C. 8 & $\begin{array}{l}\text { Substantial fuel or energy } \\
\text { consumption }\end{array}$ & 0 & 1 & 10 & 0 & 0.1 & 0 \\
\hline & Sub-total & & & & & 1 & +9.4 \\
\hline D. & Political impacts Criteria & & & & & & \\
\hline D. 1 & $\begin{array}{l}\text { Public support \& } \\
\text { participation }\end{array}$ & +0.7 & 1 & 10 & +7 & 0.1 & +0.7 \\
\hline D. 2 & Social acceptance & +0.7 & 1 & 10 & +7 & 0.1 & +0.7 \\
\hline D.3 & $\begin{array}{l}\text { Building trust in local } \\
\text { authorities }\end{array}$ & +0.8 & 1 & 10 & +8 & 0.1 & +0.8 \\
\hline D.4 & $\begin{array}{l}\text { Enhancement of } \\
\text { Governmental image }\end{array}$ & +0.8 & 1 & 10 & +8 & 0.1 & +0.8 \\
\hline D.5 & Promotion of gender equity & +0.6 & 1 & 10 & +6 & 0.1 & +0.6 \\
\hline D. 6 & $\begin{array}{l}\text { Natural infra-structural } \\
\text { plans }\end{array}$ & +5 & 1 & 10 & +50 & 0.5 & +25 \\
\hline & Sub-total & & & & & 1 & +28.6 \\
\hline & Total & & & & & & +53.23 \\
\hline
\end{tabular}


Table (3) Weighted score for environmental negative impacts in case of not applying mitigation measures in treatment (Al-Berka), (STP) units.

\begin{tabular}{|c|c|c|c|c|c|c|}
\hline Treatment units & $\begin{array}{l}\text { No. of sub. } \\
\text { Components }\end{array}$ & $\begin{array}{c}\text { Cumulative } \\
\text { Score }\end{array}$ & $\begin{array}{c}\text { Max/Min } \\
\text { Score }\end{array}$ & $\begin{array}{c}\text { Score } \\
\text { (thousands) }\end{array}$ & $\begin{array}{c}\text { Weight } \\
\text { (Thousands } \\
\text { grade) }\end{array}$ & $\begin{array}{c}\text { Normalized } \\
\text { Score \%o }\end{array}$ \\
\hline $\begin{array}{l}\text { Deceleration } \\
\text { Tanks }\end{array}$ & 8 & -81 & 800 & -101.25 & 12.5 & -1.266 \\
\hline OSpiral lift plant & 1 & -4 & 100 & -40 & 100 & -4.000 \\
\hline Bar screens & 1 & -17 & 100 & -170 & 100 & -17.000 \\
\hline $\begin{array}{c}\text { Sand separation } \\
\text { Tanks }\end{array}$ & 2 & -33 & 200 & -165 & 50 & -8.750 \\
\hline $\begin{array}{l}\text { Aeration primary } \\
\text { tanks }\end{array}$ & 2 & -33 & 200 & -165 & 50 & -8.750 \\
\hline $\begin{array}{l}\text { The chlorine } \\
\text { sterilization unit }\end{array}$ & 1 & -3 & 100 & -30 & 100 & -3.000 \\
\hline $\begin{array}{c}\text { Sludge } \\
\text { concentration } \\
\text { tanks }\end{array}$ & 2 & -40 & 200 & -200 & 50 & -10.000 \\
\hline $\begin{array}{l}\text { Sludge drying } \\
\text { unit }\end{array}$ & 3 & -35 & 300 & -117 & 33.3 & -5.005 \\
\hline $\begin{array}{l}\text { The fermented } \\
\text { sludge unit }\end{array}$ & 1 & -13 & 100 & -130 & 100 & -13.000 \\
\hline Total & & & & & & -68.925 \\
\hline
\end{tabular}

Table (4) Weighted score for environmental positive impacts in case of applying mitigation measures in treatment (Al-Berka), (STP) units.

\begin{tabular}{|c|c|c|c|c|c|c|}
\hline Treatment units & $\begin{array}{l}\text { No. of sub. } \\
\text { Components }\end{array}$ & $\begin{array}{l}\text { Cumulative } \\
\text { Score }\end{array}$ & $\begin{array}{l}\text { Max/Min } \\
\text { Score }\end{array}$ & $\begin{array}{c}\text { Score } \\
\text { (Thousands) }\end{array}$ & $\begin{array}{c}\text { Weight } \\
\text { (Thousands grade) }\end{array}$ & $\begin{array}{l}\text { Normalized } \\
\text { Score }\end{array}$ \\
\hline $\begin{array}{l}\text { Deceleration } \\
\text { Tanks }\end{array}$ & 2 & +50 & 200 & +40 & 50 & +2.000 \\
\hline Spiral lift plant & 1 & +4 & 100 & +40 & 100 & +4.000 \\
\hline Bar screens & 1 & +15 & 100 & +150 & 100 & +15.000 \\
\hline $\begin{array}{l}\text { Sand separation } \\
\text { Tanks }\end{array}$ & 2 & +32 & 200 & +160 & 50 & +8.000 \\
\hline $\begin{array}{l}\text { Aeration primary } \\
\text { tanks }\end{array}$ & 3 & +44 & 300 & +133.3 & 33.3 & +4.440 \\
\hline $\begin{array}{l}\text { The chlorine } \\
\text { sterilization unit }\end{array}$ & 9 & 209 & 900 & 232.2 & 1101 & +2.580 \\
\hline $\begin{array}{l}\text { Sludge } \\
\text { concentration } \\
\text { tanks }\end{array}$ & 1 & +24 & 100 & +41.7 & 100 & +4.170 \\
\hline Sludge drying unit & 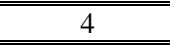 & +42 & 400 & 105 & 25 & $\begin{array}{c}+2.625 \\
\end{array}$ \\
\hline $\begin{array}{l}\text { The fermented } \\
\text { sludge unit }\end{array}$ & 1 & +24 & 100 & +240 & 100 & +24.000 \\
\hline Total & & & & & & +66.815 \\
\hline
\end{tabular}


Table (5): Resultant weighted score for environmental impacts treatment plant (AlBerka), (STP) units.

\begin{tabular}{|c|c|c|c|c|c|c|c|}
\hline 1 & 2 & 3 & 4 & 5 & 6 & 7 & 8 \\
\hline No & Plant unit & $\begin{array}{l}\text { No. of sub. } \\
\text { Components }\end{array}$ & $\begin{array}{l}\text { Cumulative } \\
\text { Score }\end{array}$ & $\begin{array}{l}\text { Max/Min } \\
\text { Score }\end{array}$ & $\begin{array}{c}\text { Score } \\
\text { (Thousands) }\end{array}$ & $\begin{array}{c}\text { Weight } \\
\text { (Thousands } \\
\text { grade) }\end{array}$ & $\begin{array}{c}\text { Normalized } \\
\text { Score \%o }\end{array}$ \\
\hline 1 & $\begin{array}{l}\text { Deceleration } \\
\text { Tanks }\end{array}$ & 10 & -31 & 1000 & -31 & 1 & -0.310 \\
\hline 2 & Spiral lift plant & 2 & 0 & 200 & 0 & 5 & 00 \\
\hline 3 & Bar screens & 2 & -2 & 200 & 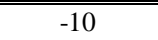 & 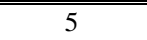 & "-0.500 \\
\hline 4 & $\begin{array}{l}\text { Sand separation } \\
\text { Tanks }\end{array}$ & 4 & -1 & 400 & -2.5 & 25 & -0.625 \\
\hline 5 & $\begin{array}{l}\text { Aeration primary } \\
\text { tanks }\end{array}$ & 5 & 11 & 500 & 22 & 200 & +0.440 \\
\hline 6 & $\begin{array}{l}\text { The chlorine } \\
\text { sterilization unit }\end{array}$ & 10 & 206 & 1000 & 2.06 & 1 & 0.206 \\
\hline 7 & $\begin{array}{l}\text { Sludge } \\
\text { concentration } \\
\text { tanks }\end{array}$ & 3 & -16 & 300 & -53.33 & 33 & -1.733 \\
\hline 8 & $\begin{array}{l}\text { Sludge } \\
\text { unit }\end{array}$ & 6 & 7 & 600 & 0.12 & 16 & 0.002 \\
\hline \multirow[t]{2}{*}{9} & $\begin{array}{l}\text { The fermented } \\
\text { sludge unit }\end{array}$ & 2 & 11 & 200 & 0.55 & 50 & 0.275 \\
\hline & & & & & & & -2.115 \\
\hline
\end{tabular}

Table (6) Weighted score for environmental impacts during construction \&

Operation phases (in case of not applying mitigation measures).

\begin{tabular}{|c|c|c|c|c|c|c|c|}
\hline 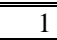 & 2 & 3 & 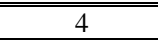 & 5 & 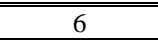 & 7 & 8 \\
\hline No. & $\begin{array}{l}\text { Environmental } \\
\text { Component }\end{array}$ & $\begin{array}{c}\text { Cumulative } \\
\text { Score }\end{array}$ & $\begin{array}{l}\text { No. of sub. } \\
\text { Components }\end{array}$ & $\begin{array}{c}\text { Max/Min } \\
\text { Score } \\
\end{array}$ & $\begin{array}{c}\text { Score } \\
\text { (Thousands) }\end{array}$ & $\begin{array}{c}\text { Weight } \\
\text { (Thousands grade) }\end{array}$ & $\begin{array}{c}\text { Normalized } \\
\text { Score \%o }\end{array}$ \\
\hline A. & $\begin{array}{l}\text { Natural resources } \\
\text { Impact criteria }\end{array}$ & & & & & & \\
\hline A.1 & Soil & $\begin{array}{l}-3.4 \\
\end{array}$ & 5 & 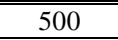 & -6.8 & 40 & -0.272 \\
\hline A. 2 & Water & $\begin{array}{l}-0.8 \\
\end{array}$ & 7 & 700 & $\begin{array}{l}-1.1 \\
\end{array}$ & 30 & -0.033 \\
\hline \multirow[t]{2}{*}{ A.3 } & Air & +3.2 & 3 & 300 & $\begin{array}{l}+10.7 \\
\end{array}$ & 30 & +0.321 \\
\hline & Sub-total & & & & & 100 & +0.016 \\
\hline B. & $\begin{array}{l}\text { Biological life } \\
\text { Impact criteria }\end{array}$ & & & & & & \\
\hline B.1 & Plant life & +4 & 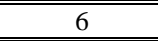 & 600 & +6.7 & 70 & +0.469 \\
\hline \multirow[t]{2}{*}{ B.2 } & Animal life & +5 & 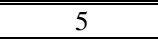 & 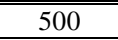 & +10 & 30 & +0.300 \\
\hline & Sub-total & & & & & 100 & +0.769 \\
\hline $\mathrm{C}$. & $\begin{array}{l}\text { Socio-economic } \\
\text { Impact criteria }\end{array}$ & & & & & & \\
\hline C.1 & $\begin{array}{ll}\text { General } \\
\end{array}$ & $\begin{array}{l}+2.5 \\
\end{array}$ & 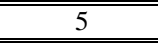 & 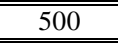 & $\begin{array}{c}+5 \\
\end{array}$ & 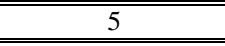 & $\begin{array}{l}+0.025 \\
\end{array}$ \\
\hline 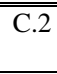 & $\begin{array}{l}\text { Public services and } \\
\text { utilities }\end{array}$ & $\begin{array}{l}-0.1 \\
\end{array}$ & 7 & 700 & 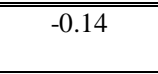 & 20 & "-0.028 \\
\hline 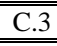 & $\begin{array}{l}\text { Human health } \\
\end{array}$ & $\begin{array}{c}-0.1 \\
\end{array}$ & 4 & 400 & $\begin{array}{c}-0.25 \\
\end{array}$ & 20 & $\begin{array}{c}-0.005 \\
\end{array}$ \\
\hline$\overline{C .4}$ & $\begin{array}{l}\text { Creation of new } \\
\text { Communities }\end{array}$ & +2 & 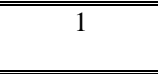 & 100 & +20 & 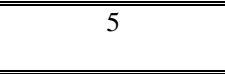 & +0.100 \\
\hline C.5 & $\begin{array}{l}\text { Solid waste \& } \\
\text { Hazardous waste }\end{array}$ & -2 & 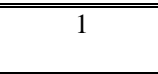 & 100 & 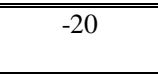 & 10 & "-0.200 \\
\hline C.6 & $\begin{array}{l}\text { NGOs and local } \\
\text { Unions }\end{array}$ & 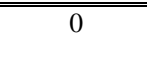 & 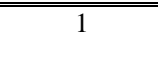 & 100 & 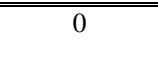 & 10 & 0.000 \\
\hline
\end{tabular}


Contend

\begin{tabular}{|c|c|c|c|c|c|c|c|}
\hline C.7 & $\begin{array}{l}\text { Sustainability of } \\
\text { Services }\end{array}$ & 0 & 1 & 100 & 0 & 0.2 & 0 \\
\hline \multirow[t]{2}{*}{ C.8 } & $\begin{array}{l}\text { Substantial fuel } \\
\text { or energy consumption }\end{array}$ & -5 & 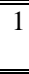 & 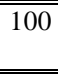 & -50 & 10 & "-0.500 \\
\hline & Sub-total & & & & & 100 & -0.583 \\
\hline D. & $\begin{array}{l}\text { Political impacts } \\
\text { Criteria }\end{array}$ & & & & & & \\
\hline D. 1 & $\begin{array}{l}\text { Public support \& } \\
\text { Participation }\end{array}$ & +0.6 & 1 & 100 & +6 & 10 & +0.060 \\
\hline D.2 & Social acceptance & +0.6 & 1 & 100 & +6 & 10 & +0.060 \\
\hline D.3 & $\begin{array}{l}\text { Building trust in local } \\
\text { authorities }\end{array}$ & +0.6 & 1 & 100 & +6 & 10 & +0.060 \\
\hline D. 4 & $\begin{array}{l}\text { Enhancement of } \\
\text { Governmental Image }\end{array}$ & +0.6 & 1 & 100 & +6 & 10 & +0.060 \\
\hline D.5 & $\begin{array}{l}\text { Promotion of } \\
\text { Gender equity } \\
\end{array}$ & 0 & 1 & 100 & 0 & 10 & 0 \\
\hline \multirow[t]{3}{*}{ D.6 } & $\begin{array}{l}\text { Natural infra- } \\
\text { structural plans }\end{array}$ & +5 & 1 & 100 & +50 & 50 & 2.500 \\
\hline & Sub-total & & & & & 100 & +2.740 \\
\hline & Total & & & & & & +2.942 \\
\hline
\end{tabular}

Table (7) Weighted score for environmental impacts during construction \& Operation phases (in case of applying mitigation measures).

\begin{tabular}{|c|c|c|c|c|c|c|c|}
\hline 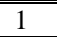 & 2 & 3 & 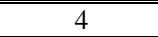 & $\overline{c 5}$ & 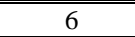 & 7 & 88 \\
\hline No. & Environmental Component & $\begin{array}{c}\text { Cumulative } \\
\text { Score }\end{array}$ & $\begin{array}{l}\text { No. of sub. } \\
\text { Components }\end{array}$ & $\begin{array}{c}\text { Max/Min } \\
\text { Score }\end{array}$ & $\begin{array}{c}\text { Score } \\
\text { Thousands }\end{array}$ & $\begin{array}{c}\text { Weight } \\
\text { (Thousands } \\
\text { grade) }\end{array}$ & $\begin{array}{c}\text { Normalized } \\
\text { Score }\end{array}$ \\
\hline A. & Natural resources Impact criteria & & & & & & \\
\hline A.1 & Soil & +1.35 & 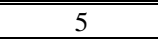 & 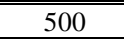 & +2.7 & 40 & +0.108 \\
\hline A.2 & Water & +1.75 & 7 & 700 & +2.5 & 30 & +0.075 \\
\hline A.3 & Air & +5.6 & 3 & 300 & +18.7 & 30 & +0.560 \\
\hline & Sub-total & & & & & 100 & +0.743 \\
\hline B. & "Biological life Impact criteria & & & & & & \\
\hline B.1 & Plant life & +3.45 & 6 & 600 & +5.75 & 70 & +0.403 \\
\hline B.2 & Animal life & +7.2 & 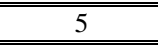 & 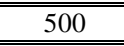 & +14.4 & 30 & +0.432 \\
\hline & Sub-total & & & & & 100 & +0.835 \\
\hline$\overline{\mathrm{C}}$. & Socio-economic Impact criteria & & & & & & \\
\hline C.1 & General & +7 & 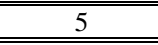 & 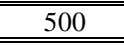 & +14 & 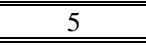 & +0.070 \\
\hline C.2 & Public services and utilities & +0.2 & 7 & 700 & +0.2857 & 20 & +0.060 \\
\hline 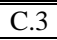 & Human health & +2.2 & 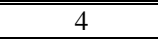 & 400 & +5.5 & 20 & +0.110 \\
\hline C.4 & Creation of new Communities & +4 & 1 & 100 & +40 & $\overline{5}$ & +0.200 \\
\hline$\overline{C C .5}$ & Solid waste \& Hazardous waste & +3 & 1 & 100 & +30 & 10 & +300 \\
\hline C.6 & NGOs and local Unions & +2 & 1 & 100 & +20 & 10 & +0.200 \\
\hline$\overline{~ C .7}$ & Sustainability of Services & $\overline{00}$ & 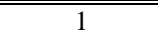 & 100 & 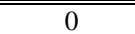 & 20 & 0.0 \\
\hline
\end{tabular}

\begin{tabular}{||l|l|c|c|c|c|c|c||}
\hline \hline C.8 & $\begin{array}{l}\text { Substantial fuel or energy } \\
\text { consumption }\end{array}$ & 0 & 1 & 100 & 0 & 10 & 0 \\
\hline & Sub-total & & & & & 100 & +0.940 \\
\hline D. & Political impacts Criteria & & & & & & \\
\hline D.1 & Public support \& participation & +0.7 & 1 & 100 & +7 & 10 & +0.070 \\
\hline D.2 & Social acceptance & +0.7 & 1 & 100 & +7 & 10 & +0.070 \\
\hline D.3 & Building trust in local authorities & +0.8 & 1 & 100 & +8 & 10 & +0.080 \\
\hline D.4 & $\begin{array}{l}\text { Enhancement of Governmental } \\
\text { image }\end{array}$ & +0.8 & 1 & 100 & +8 & 10 & +0.080 \\
\hline D.5 & Promotion of gender equity & +0.6 & 1 & 100 & +6 & 10 & +0.060 \\
\hline D.6 & Natural infra-structural plans & +5 & 1 & 100 & +50 & 50 & +2.500 \\
\hline & Sub-total & & & & & & +2.860 \\
\hline & Total & & & & +5.323 \\
\hline
\end{tabular}


-Cell 1: number of items.

-Cell 2: environmental components (A, B, etc.) and sub-components (A.1, A.2, etc.).

-Cell 3: number of sub-components in each component.

-Cell 4: cumulative score which represents the sum of scores of sub-items

ranging from $(-10)$ to $(+10)$ of ten-grade system and(-1000)to(+1000) of thousand system.

-Cell 5: Max. / Min. score, which is (10) for all items multiplied by number of sub-components.

-Cell 6: the result of dividing cell 3 over cell 5, then multiplying the result times 100.

-Cell 7: weight of each sub-component as part of the main component.

-Cell 8: the result of multiplying cell 6 times cell 7.

The construction activities considered in the study are:

1- Occupation of land.2- Site preparation and irrigation pipe lines.3- Transportation of materials to the site.4- Materials handling and storage.5- Construction activities.

- The operation activities considered in the study are:

1- Treatment of waste water.2- Irrigation pump station and wood forest.3- O (operation) \& M (maintenance) of the treatment plant.4- Filtration. 5- Pumps and irrigation network. 6. Handling of sludge.

Table (1) summerizes the positive and negative effect of AL-Berka

Table (2) presents the weighted score for environmental impacts. Environmental impact system used of the expected situation assessment in the future for the environmental quality can be represented by the following equation:

$\mathrm{Er}=$ (VI) $1 \mathrm{WI} /$ (VI) $2 \mathrm{WI}$

Er: Environmental impact value.

(VI) 1: Environmental impact value during project construction.

(VI) 2: Environmental impact value during project operation.

$\mathrm{WI}=$ The importance (weight) the relativity for the criterion.

$\mathrm{R}$ : Criteria number summations.

Analysis method application:

1-Deceleration Tanks:

-Negative: Conveys the dismissal in an exposed aqueduct for a distance what avert for the pollution increase causes odor harm in the health and the methane gases spread and the ammonia and the hydrogen sulfur And the bacteria activates.

A classification Batil of weighted environmental for the development projects the natural resources.

-The odor and the visual factors:

$$
\begin{array}{r}
-3 \times 100 \%=-3 \\
-4 \times 100 \%=-4 ; \\
-10 \times 100 \%=-10 \\
-16 \times 100 \%=-16 \\
-16 \times 100 \%=-16
\end{array}
$$$$
\text { -Noises: }
$$

-The water appearance:

-The general shape for the water:

The odor and the floating materials: 
-A water surface scope:

$-10 \times 10 \%=-10$

-Gas emissions:

-The moved local diseases in the water;

Total:

$-17 \times 100 \%=-17$

$-81$

-Positive: Execution of carbon pumping project helps on oxidization results in light gasses adsorption.

-active carbon:

$+22 \times 100 \%=+22$;

-flow change:

$+28 \times 100 \%=+28$;

Total:

-Enhancement:

2- Sludge concentration tanks:

Negative: Causes diseases among workers emission and odor

-The active sludge due to activation bacteria:

Total:

$$
\begin{aligned}
+ & +50 \\
= & -31 \%
\end{aligned}
$$

Positive: Reducing of the volume of both water and sludge and pumping tanks drying solar.

$+24 \times 100=+24$

Enhancement:

$$
-40 /+24=-1.66
$$

\section{Details calculation for project environmental impacts:}

$-17 \times 100=-17$

$-23 \times 100=-23$;

$=-40$;

In case of not applying mitigation measures during construction \& operation: depending on Analytical Hierarch Process, (Weights) Factors influencing the choice of the optimal alternative to sewage treatment plants.

A) Natural Resources Impacts Criteria:

A.1.Soil: investigation report performed at location of the treatment plant shows that the main feature of the soil is the sand layers and ground water. (At level.)

A.1.1 Soil erosion

A.1.2 Soil fertility

A.1.3 Soil salinity

A.1.4 Preventing soil pollution

A.1.5 Ground contours

Total

A.2.Water

A.2.1

A.2.2

A. 2.3

A.2.4

A.2.5

A.2.6

A.2.7

Total
Irrigation water quality

Irrigation water quantity

Drainage water quality

Drainage water quantity

Ground water level

Ground water quantity

Ground water quality
$+2 \times 15 \%=+0.3$ $-5 \times 30 \%=-1.5$;

$0 \times 5 \%=0$;

$-5 \times 40 \%=-2$;

$-2 \times 10 \%=-0.2$;

-3.4 ;
$-5 \times 15 \%=-0.75$;

$+5 \times 15 \%=+0.75$

$-5 \times 10 \%=-0.5$;

$0 \times 10 \%=0$;

$0 \times 20 \%=0$;

$0 \times 15 \%=0$;

$-2 \times 15 \%=-0.3$; 
A.3.Air

A.3.1 Gas emissions

$5 \times 40 \%=+2$

A.3.2 Dust pollution

$2 \times 50 \%=+1$;

A.3.3 Local climate

$+2 \times 10 \%=0.2$;

Total

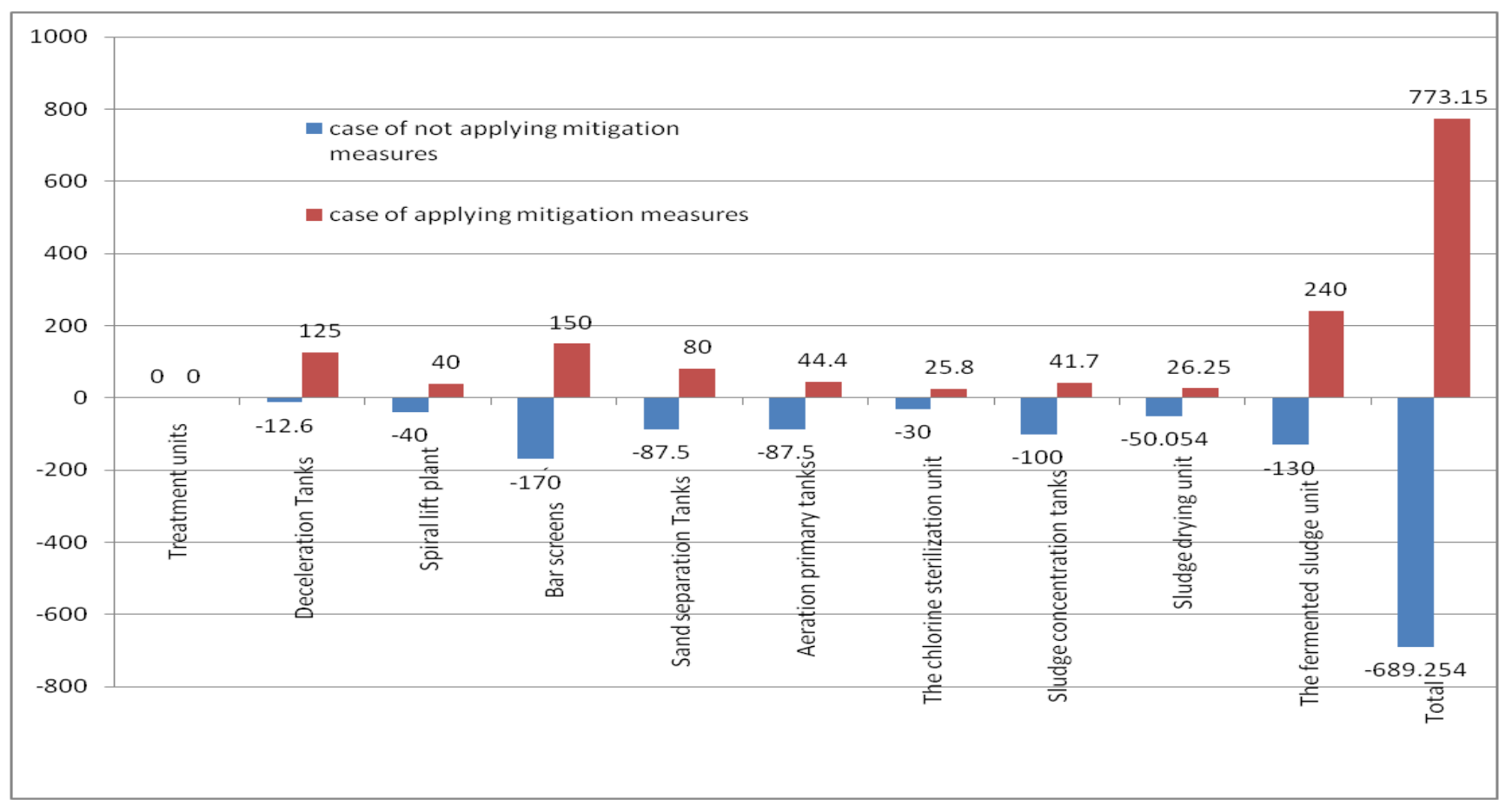

Figure (1) shown comparison in case not applying and applying mitigation measures.

Table (8) Comparison Test 10th to 1000th Of AL-BERKA (Odor, Sludge).

\begin{tabular}{|c|c|c|c|c|}
\hline $\mathrm{NO}$ & \multicolumn{2}{|c|}{$10^{\mathrm{th}}$} & \multicolumn{2}{|c|}{$1000^{\text {th }}$} \\
\hline & Before mitigation & After mitigation & Before mitigation & After mitigation \\
\hline$\overline{\text { Odor control }}$ & $-12.6 \%$ & $+20 \%$ & $-1.260 \%$ & $+2.000 \%$ \\
\hline Sludge & $-50.05 \%$ & $+26.25 \%$ & $-5.005 \%$ & $+2.625 \%$ \\
\hline
\end{tabular}

\section{Conclusions:}

1.The assessment of AL-BERKA treatment plant shows that it has a Weight of (+29.42\% ten-grade system), (+2942 thousands-grade system) in case of not applying mitigation measures. The measure enhanced to (53.23\% ten-grade system), (5323 thousands grade system) in case of applying mitigation measure giving a resultant 
enhancement about $81 \%$.

2.Odor represents one of the most environmental aspects that has negative impact on the environment and the mitigation applied by pumping carbon in the plant site gave an enhancement[weight before mitigation(-81\% ten-grade system), $(-8.100 \%$ thousands grade system)] and [weight after mitigation (+50\% ten-grade system), $(+5.000 \%$ )thousands grade system)] enhancement about $+62 \%$.

3.Sludge generated from treatment process has a significant negative impact on the environmental (weight before mitigation $-48 \%$ ). This causes environmental problems to air soil, groundwater, surface water and public health. The mitigation gave an enhancement(weight after mitigation $+88 \%$ ) enhancement about $+84 \%$.

4.An improvement of $(-5.83 \sim+9.4 \%$ tengrade system, $+0.940 \%$ thousands system $)$ above baseline conditions, is considered as remarkable positive impact on the socioeconomic dimension enhancement about $+61 \%$.

5.A remarkable improvement on the political impact dimension scores $(+27.4 \sim 28.6 \%$ above baseline condition. The main reasons are the social acceptance of the project and the enhancement of government image as a result of implementing the project as well as the willingness to apply mitigation measures in the long run enhancement about $+44 \%$.

6.The suggested one thousands weight measure for impact enhanced analysis accuracy and made comparisons and decision more easier than tenth grade evaluation.

7.The water quality enhancement produced from treated process.

8.The sludge quality enhancement produced from treated process.

9.Improvement of the environmental monitoring plan for reusing treated water in irrigation and agriculture.

10. Improvement the environmental monitoring plan for reusing sludge, produced by treating process, in fertilization.

11.Necessity of finding good environmental solutions to get over odors produced by treating water and sludge.

12.Recommendation in choosing sites of treatment plant must be distant from Residential areas.

\section{References:}

[1]Oswald, W.J. and Golueke, C.G. (1968): Large scale production of algae. In: R.I. Mteries and S.R. Tannenbaum (Eds.), Single cell protein. Mlt Press, Cambridge, Massachusetts and London, England.

[2] Matusiak, K.; Chrost, R. and krzywicka, A. (1971): Bacterial microflora in Chlorella vulgaris culture. Acta Microbiologica Polonica, 3 (20D): 189-193.

[3]Shelef, G.; Azov, Y.; Moraine, R. and Oran, G. (1980): Algal mass production as an integral part of a wastewater treatment and reclamation system. In: Algal Biomass Ed. G. Shelf and C.J. Soeder .Elsevier / North - Holland Biomedical Press, Amsterdam, pp. 163. 
[4]Rachlin, J. W., Jensen, T.E. And Warkentine, B. (1983): The growth response of the Diatom Navicula incerta to selected concentrations of the metals: cadmium , copper, lead and zinc . Bull. Torret. Bot. Club. 110: 217-223.

[5]WPCF (1987) observed that the temperature affects the solubility limitation of oxygen required for aerobic treatment of wastewater.

\section{Nomenclatures:}

TSS Total Suspend Ended Solids.

WWTP Waste Water Treatment Plant.

COD Chemical Oxygen Demand $[\mathrm{m} / \mathrm{L}]$.

$\mathrm{BOD}_{5} \quad$ Biological Oxygen Demand $[\mathrm{m} / \mathrm{L}]$.

EIA Environment Impact Assessment. 